\title{
Validation of taste-tests conducted by mother-infant dyads in the home environment to assess infant taste preferences
}

\author{
J.E. Miller and J.M. Wallace \\ The Rowett Institute, University of Aberdeen, Aberdeen, AB25 2ZD
}

Studies investigating infant taste preferences require a reliable measure of taste acceptance. Previously, taste-tests have been conducted in controlled laboratory conditions, usually several months after weaning has started ${ }^{(1,2)}$. This approach is burdensome for participants and the unfamiliar environment may influence appetitive behaviour. This study aims to determine whether reliable data can be extracted from taste-tests conducted by mother-infant dyads, independently, in their own homes when weaning commences.

Twenty-six infants began weaning at $24.8 \pm 0.3$ (mean \pm sem) weeks old. Potato puree was offered first until they were competent at eating, then four taste-tests were conducted using potato, apple, broccoli and spinach. The importance of a consistent approach was emphasised to the mothers and they were asked to weigh the amount of puree consumed and rate enjoyment using a Likert scale. The first ten spoonfuls of each video were observed by two independent assessors and the behaviours ( 3 negative/1 positive), facial expressions (3 negative) and rate of acceptance (early/late/enforced/rejected) were scored using The Feeding Infants: Behaviour and Facial Expression Coding System (FIBFECS ${ }^{3}$ ) - a validated method of assessing infants' wanting and liking of food. Spearman's rho correlations between average FIBFECS scores, amount of puree eaten and mothers' ratings of liking were conducted using IBM SPSS Statistics version 25 .

Intake of all purees was inversely associated with negative behaviours $(\mathrm{r}=-0.75-0.49, \mathrm{P} \leq 0.01)$, all behaviours combined $(\mathrm{r}=-0.61-0.49, \mathrm{P} \leq 0.02)$, negative expressions $(\mathrm{r}=-0.67-0.43, \mathrm{P} \leq 0.03)$ and overall disliking score (expressions and behaviours combined; $r=-0.74-0.46, \mathrm{P} \leq 0.02)$; and it was positively associated with rate of acceptance $(\mathrm{r}=0.41-0.62, \mathrm{P} \leq 0.04)$. Liking as rated by mothers correlated positively with intake $(\mathrm{r}=0.49-0.68, \mathrm{P} \leq 0.01)$ and negatively with overall disliking score $(\mathrm{r}=-0.54-0.4, \mathrm{P}<$ $0.05)$ for all purees except potato $(\mathrm{P}<0.05)$. The rate of acceptance for all purees was inversely associated with negative behaviours $(\mathrm{r}=-0.83-0.56, \mathrm{P} \leq 0.003)$ and all behaviours combined $(\mathrm{r}=-0.814-0.41, \mathrm{P} \leq 0.04)$, and with negative expressions for plain and apple purees only $(\mathrm{r}=-0.43-0.39, \mathrm{P}<0.05)$. Negative behaviours and expressions were positively associated for broccoli and spinach purees $(\mathrm{r}=0.47-0.63, \mathrm{P} \leq 0.02)$.

Puree intake related well to all other aspects and provides a reliable objective measure of liking i.e. the more a food is liked, the more is eaten. Mothers' ratings of liking corresponded with overall disliking scores, however, not with behaviours or expressions on their own. Behaviours/expressions are subtle and may be missed by mothers thus close, independent observation via the FIBFECS method is required. Furthermore, a mother's subjective rating may be influenced by factors such as their baby's temperament. The three FIBFECS measures (behaviours, expressions and acceptance) related well, however, stronger relationships existed for behaviours and expressions combined than expressions alone. These results closely agree with Nekitsing ${ }^{(3)}$ and validate the reliability of data produced by mother-infant dyads in their own homes to assess infants' taste preferences at the outset of weaning.

1. Hausner H, Nicklaus S, Issanchou S et al. (2010) Clin Nutr 29, 1, 141-8.

2. Mennella JA, Daniels LM \& Reiter AR (2017) Am J Clin Nutr 106, 1, 67-76.

3. Nekitsing C, Madrelle J, Barends C et al. (2016) Food Qual. Prefer 48, $228-237$. 